\title{
An Organic Intravesical Foreign Body Caused by Penetrating Trauma that was Missed during Initial Management
}

\author{
Hoon Ah Jang, Sung Gu Kang, Young Hwii Ko, Seok Ho Kang, Jun Cheon, Je Jong Kim, Jeong Gu Lee \\ Department of Urology, Korea University Anam Hospital, Seoul, Korea
}

\begin{abstract}
We report a case of an intravesical foreign body that was incompletely removed endoscopically and that defied diagnosis with current diagnostic tools. A 65-year-old man visited Korea University Anam Hospital complaining of dysuria and a sensation of residual urine. His medical history included an intravesical foreign body caused by penetrating trauma, and he had undergone endoscopic removal of foreign bodies 1 year previously. After additional remnant intravesical foreign bodies were found, he had undergone additional endoscopic removal and his urinary symptoms subsided. After 2 years, however, he again presented to the clinic complaining of dysuria and gross hematuria. Cystoscopy and computed tomography for intravesical foreign bodies were performed, but no evidence of a remnant foreign body was found. Open exploration revealed a remnant foreign body penetrating the bladder. A partial cystectomy including the foreign body was performed. We suggest that cases of penetrating injury with a radiolucent object may warrant primary open exploration and foreign body removal owing to the inherent difficulties in diagnosis and endoscopic treatment of such objects.
\end{abstract}

Keywords: Urinary bladder; Foreign bodies; Penetrating wounds

Various foreign bodies have been observed in the urinary bladder [1], including pieces of urethral catheter, wires, intrauterine contraceptive devices (IUDs), surgical staples lodged in kidney stones, chicken bones, thermometers, encrusted sutures, bullets, gauze, pencils, broken pieces of endoscopic instruments, and other items. The routes of entry into the bladder include penetrating trauma, migration from neighboring organs, iatrogenic introduction, and self-insertion for sexual gratification [2-4]. Cystoscopy is rarely required to diagnose foreign objects in the bladder, because radiopaque objects are visible on radiographs and others are visible on ultrasound. However, organic foreign bodies such as wood or seeds are difficult to identify with these methods, especially when small or thin. Most foreign bodies can be retrieved endoscopically, and open surgery is usually not required [3]. In this report, we present a case of an organic foreign body introduced by penetrating trauma that was missed during the initial management of the patient.

\section{CASE REPORT}

A 65-year-old man presented to Korea University Anam Hospital complaining of dysuria and the sensation of incomplete emptying. His symptoms had begun several months previously and had not improved with antibiotic treatment. His medical history included a fall 1 year previously onto bamboo branches, which had penetrated his right perianal area. At that time, treatment including endoscopic removal of the bamboo splinters and closure of the wound was performed. On physical examination, an indistinct scar was observed in the right perianal area and the wound was clean. Laboratory examination revealed copious red and white blood cells in the urine; the urine culture grew no organisms. Plain X-ray and intravenous pyelography showed distortion of the bladder and normal urine drainage in both ureters (Fig. 1A), and abdominal sonography showed a perivesical mass on the right lateral bladder wall. Under general
Corresponding author: Jeong Gu Lee

Department of Urology, Korea University Anam Hospital, 73 Inchon-ro, Seongbuk-gu, Seoul 136-705, Korea

Tel: +82-2-920-5683 / Fax: +82-2-928-7864 / E-mail: jeongkl@kumc.or.kr Submitted: October 16, 2011 / Accepted after revision: September 24, 2012
This is an Open Access article distributed under the terms of the Creative Commons Attribution Non-Commercial License (http://creativecommons.org/licenses/by-nc/3.0/) which permits unrestricted non-commercial use, distribution, and reproduction in any medium, provided the original work is properly cited. 
anesthesia, transurethral cystoscopy showed erythema and ulceration of the right lateral bladder mucosa with a foreign body embedded in granulation tissue (Fig. 1B). The foreign body was removed with cystoscopic forceps and was identified as bamboo (size $1 \times 0.4 \mathrm{~cm}$ ). The Foley catheter remained in place for 1 week, after which the patient was discharged.

The patient returned for follow-up 4 weeks postoperatively without urinary symptoms. Two years later, however, the patient presented to the clinic complaining of dysuria and gross hematuria. Red and white blood cells were abundant in the urine sedimentation. Cystoscopy revealed an erythematous and swollen bladder wall at the site of the previous operation, without a visible foreign body (Fig. 2A). Computed tomography (CT) showed right lateral bladder wall thickening with perivesical space infiltration and reactive pelvic lymphadenopathy. We were suspicious of remnant foreign bodies and decided to perform an open exploration under general anesthesia (Fig. 2B).

A low midline incision was made, revealing thickening of the right bladder wall with diffuse adhesions between the bladder and the pelvic wall. During adhesiolysis, a tract entering the bladder was identified. After opening the bladder dome, we could see that the tract was connected to the bladder cavity and contained foreign bodies. The foreign bodies were buried by extremely swollen bladder mucosa, which may have prevented their detection by cystoscopy. The tract extended across the pelvic floor laterally below the ischial bone. We completely excised the inflammatory tissue, including the foreign bodies and the diseased bladder wall (Fig. 3). On the seventh postoperative day, the urethral catheter was removed and the patient was discharged. At the 3-month follow-up, the patient was asymptomatic, was voiding normally, and had no evidence of urinary tract infection.

\section{DISCUSSION}

Intravesical foreign bodies are an important consideration in the differential diagnosis of lower urinary tract problems $[1,5]$. The routes of entry into the bladder include penetrating trauma, migration from neighboring organs, iatrogenic introduction, and self-insertion for sexual gratification. Transurethral introduction of foreign bodies is common and is usually selfinflicted or iatrogenic. Examples of foreign objects introduced transurethrally include electric wire, batteries, magnets, urethral catheters, the beak of the resectoscope sheath, and thermometers. Although foreign bodies rarely migrate into the urinary bladder from adjacent organs, objects have been known to perforate the urinary bladder from the gastrointestinal or female genital tracts, sometimes accompanied by a fistula. Perforating objects have included IUDs, a vaginal pessary, prosthetic slings, surgical gauze, nonabsorbable sutures, surgical clips, chicken bones, and fish bones. Intravesical foreign objects can remain asymptomatic for long periods of time. For example, Dietrick et al. [6] reported a case of IUD migration into the bladder that remained asymptomatic for 16 years. Penetrating
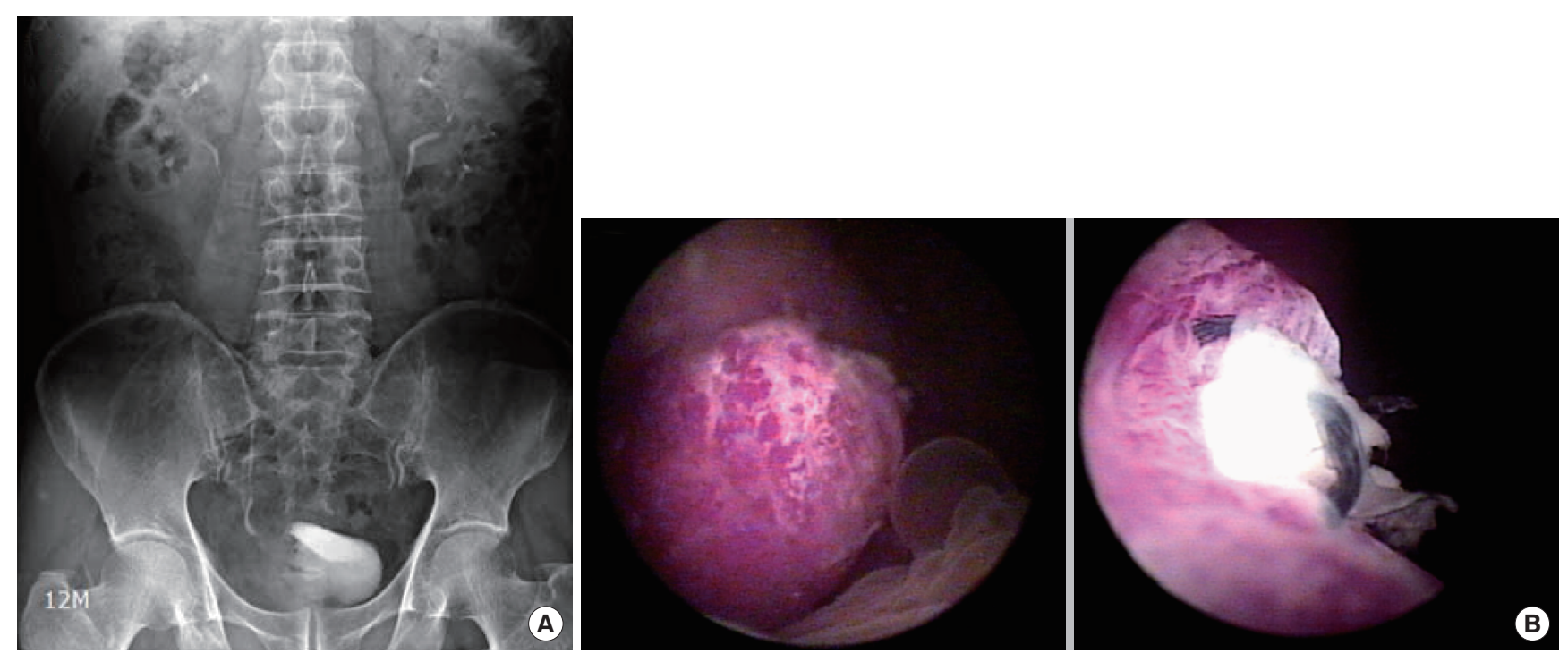

Fig. 1. (A) Intravenous pyelography showed a filling defect on the right side of the bladder. (B) Cystoscopy showed erythema of the right lateral bladder mucosa with a large granulomatous mass. The foreign body was embedded in the granulation tissue. 

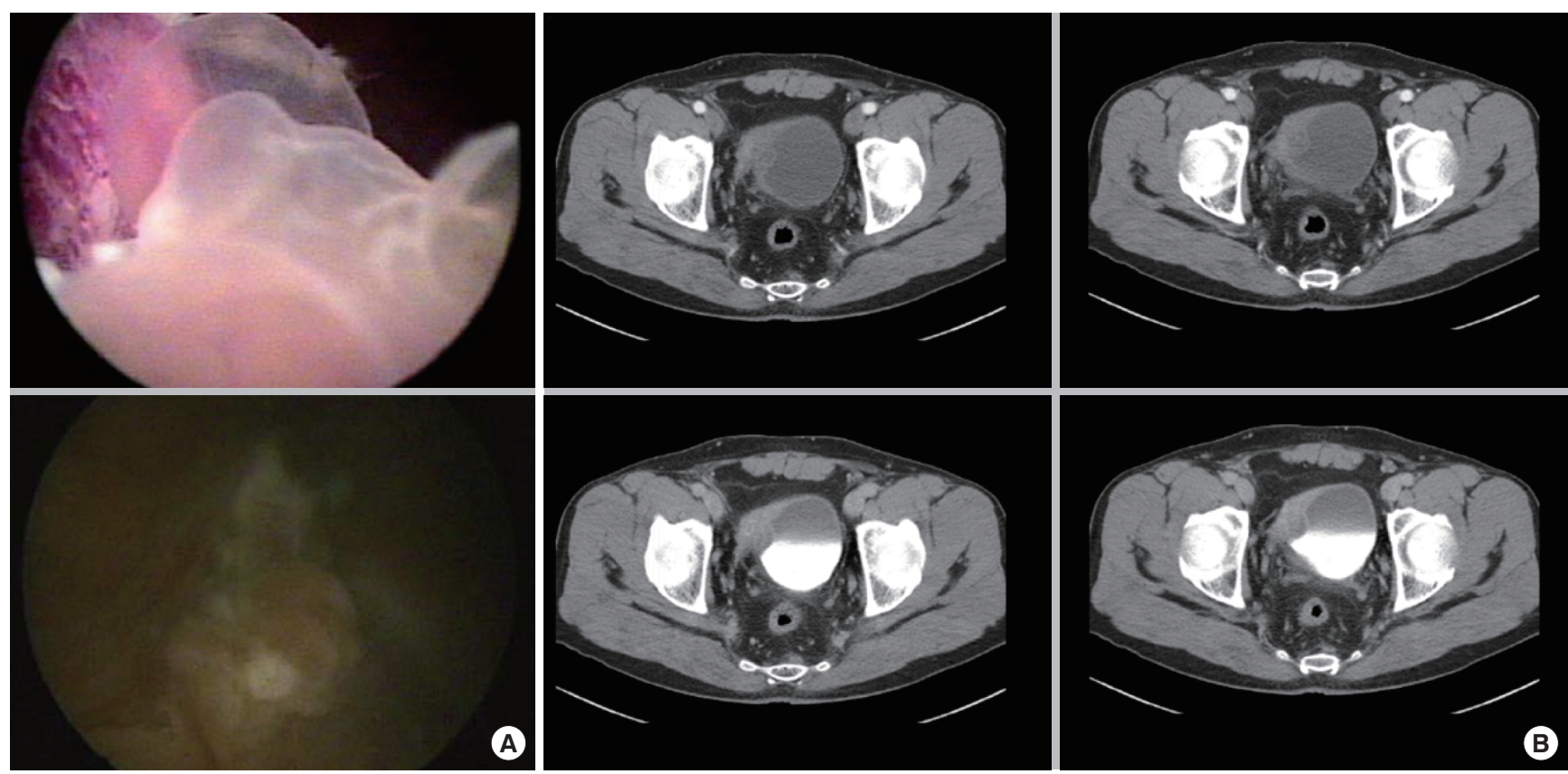

Fig. 2. (A) Cystoscopy showed erythema and swelling of the bladder wall at the previous operation site, but a foreign body was not observed. (B) The computed tomography scan showed right lateral bladder wall thickening with perivesical space infiltration and reactive pelvic lymphadenopathy. These findings were suspicious for remnant foreign bodies, which led us to perform open surgical exploration.

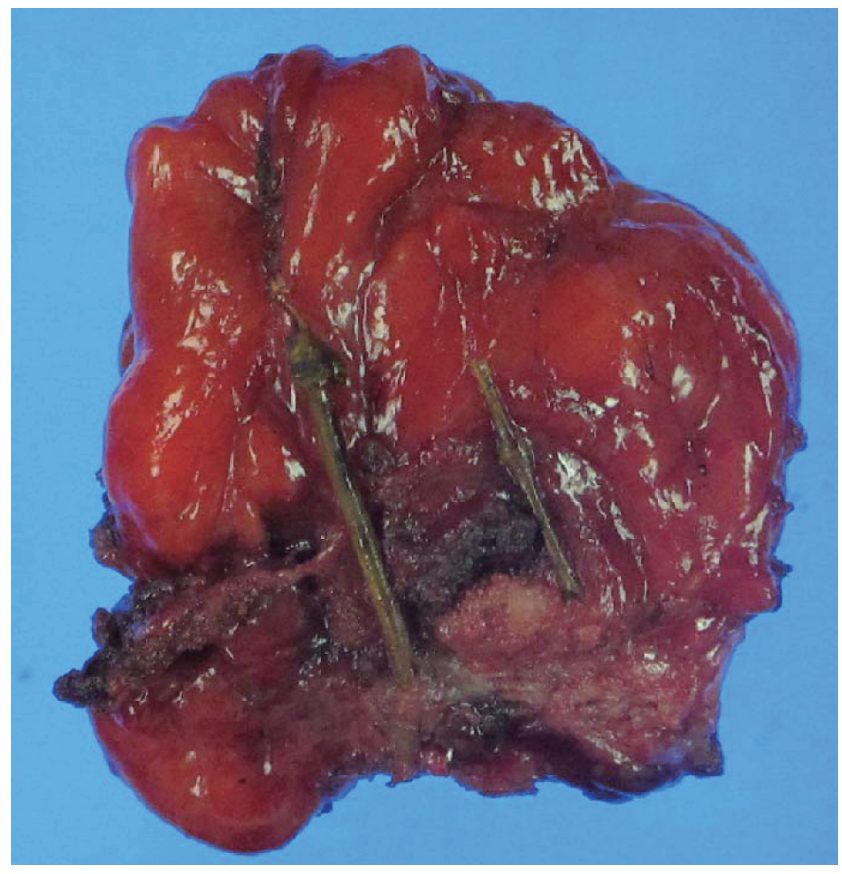

Fig. 3. Gross photography of the bamboo splinters in the bladder wall.

trauma resulting in an intravesical foreign body is relatively rare; several reports have identified objects such as bullets and wood. The route may be transabdominal, transperineal, or transrectal. In these cases, the intravesical foreign bodies were discovered immediately and were removed through the urethra by cystoscopy. Several unusual cases of intravesical foreign bodies have been reported, including a bunch of hair from a perforated ovarian dermoid cyst and skeletal remnants from an extrauterine pregnancy.

Symptoms of intravesical foreign bodies are similar to those of acute cystitis, such as dysuria, frequency, hematuria, and occasionally acute urinary retention. On the other hand, patients may be asymptomatic or present with only minimal discomfort. Clinicians should take a detailed medical history, including discussion of the patient's sexual practices and previous bladder or pelvic organ surgery, and perform a thorough physical examination that includes genital and rectal examinations.

The diagnosis of intravesical foreign bodies may include Xray, ultrasonography, and cystoscopy. Whereas radiopaque foreign bodies are visible on X-ray, intravenous urography or retrograde urethrography may occasionally detect radiolucent objects and can provide additional information [1]. The vast majority of organic foreign bodies are radiolucent, such as wood, insects, and hair; even animal bones are not always visible owing to their small size. Ultrasonography is a valuable diagnostic 
tool for radiolucent foreign bodies [7]. A foreign body's echogenicity and ultrasonographic appearance vary according to its shape and the acoustic impedance between it and the surrounding tissue. Cystoscopy can confirm the presence of an intravesical foreign body; identify its type, location, and size; and be used to perform definitive treatment. CT and magnetic resonance imaging (MRI) are useful for objects not visible on X-ray or cystoscopy, as well as to exclude other diagnoses. However, the effectiveness of CT and MRI for diagnosing intravesical foreign bodies is still under evaluation.

The standard initial management of intravesical foreign bodies is endoscopic removal. Most foreign bodies in the bladder can be successfully removed endoscopically by using grasping forceps, a retrieval basket, or a cutting loop [3]. Minimally invasive procedures such as endoscopic management are usually successful and minimize bladder and urethral injuries. If cystoscopic removal fails, however, an open procedure such as perineal urethrotomy or suprapubic cystostomy is performed. Various alternative methods of removal have been described, including meatotomy, internal or external urethrotomy, Fogarty catheterization, and injection of solvents. Recently, several minimally invasive alternative approaches have been reported. Jung et al. [8] reported the laparoscopic-assisted removal of an intravesical foreign body, Habermacher and Nadler [9] reported the use of a holmium laser to fragment a foreign body before successful removal, and Ko et al. [10] introduced laparoscopic-assisted removal of intravesical foreign bodies by use of a pneumovesicum method.

In general, intravesical foreign bodies can be diagnosed by simple X-ray and cystoscopy and can be removed either endoscopically or by open cystostomy depending on size. In our case, the bamboo branches that penetrated the pelvic floor through the perianal area and pierced the bladder were initially treated endoscopically, but they were not completely removed and remained in the perivesical area for several months. The remnant fragments eventually led to chronic inflammation and migrated into the bladder, causing a recurrence of urinary symptoms. Organic foreign bodies such as wood cause a stronger inflammatory response than do inorganic materials, which in this case resulted in our patient's swollen bladder wall and significant inflammatory reaction. Because wood is radiolucent, it was not visible on X-ray. In addition, the CT scan failed to detect the foreign body because of the severe inflammation and reactive tissue granulation at the site. Given our experience, we suggest that cases of penetrating injury with a radiolucent object may warrant primary open exploration and foreign body removal owing to the inherent difficulties in diagnosis and endoscopic treatment.

\section{CONFLICT OF INTEREST}

No potential conflict of interest relevant to this article was reported.

\section{REFERENCES}

1. Eckford SD, Persad RA, Brewster SF, Gingell JC. Intravesical foreign bodies: five-year review. Br J Urol 1992;69:41-5.

2. Datta B, Ghosh M, Biswas S. Foreign bodies in urinary bladders. Saudi J Kidney Dis Transpl 2011;22:302-5.

3. Mannan A, Anwar S, Qayyum A, Tasneem RA. Foreign bodies in the urinary bladder and their management: a Pakistani experience. Singapore Med J 2011;52:24-8.

4. Moon SJ, Kim DH, Chung JH, Jo JK, Son YW, Choi HY, et al. Unusual foreign bodies in the urinary bladder and urethra due to autoerotism. Int Neurourol J 2010;14:186-9.

5. Rafique M. Intravesical foreign bodies: review and current management strategies. Urol J 2008;5:223-31.

6. Dietrick DD, Issa MM, Kabalin JN, Bassett JB. Intravesical migration of intrauterine device. J Urol 1992;147:132-4.

7. Barzilai M, Cohen I, Stein A. Sonographic detection of a foreign body in the urethra and urinary bladder. Urol Int 2000;64:178-80.

8. Jung US, Lee JH, Kyung MS, Kim KH, Choi JS. Laparoscopic removal of an intravesical foreign body after laparoscopically assisted vaginal hysterectomy: a case report and review of the literatures. Surg Laparosc Endosc Percutan Tech 2008;18:420-2.

9. Habermacher G, Nadler RB. Intravesical holmium laser fragmentation and removal of detached resectoscope sheath tip. J Urol 2005; 174(4 Pt 1):1296-7.

10. Ko YH, Kang SG, Kang SH, Park HS, Lee JG, Kim JJ, et al. Removal of long, complex foreign bodies from bladder using single laparoscopic port under pneumovesicum. J Laparoendosc Adv Surg Tech A 2010;20:639-42. 